\title{
COVID-19 and Promising Expected Vaccines
}

\author{
Ebtehal M Fawzi ${ }^{1}$, Sheima A Elbasheer ${ }^{2 *}$, Alaa A Elhussein ${ }^{3}$, Ahmed 0 Abuelhassan ${ }^{1}$, Abdelmonem M \\ Ali $^{1}$, Alfatih Aboalbasher Yousif ${ }^{2,4}$, Nagia S Ahmed ${ }^{5}$ and Mayada Kh Ali ${ }^{6}$ \\ ${ }^{1}$ Department of Clinical Chemistry, College of Medical laboratory science, AL- Neelain University, Sudan \\ ${ }^{2}$ Department of Microbiology, College of Medical laboratory science, University of Alzaiem Al Azhari, Sudan \\ ${ }^{3}$ Department of Hematology and Immunohematology, Sudan University of Science and Technology, Sudan \\ ${ }^{4}$ Department of Pathology, Ibra Hospital, Ministry of Health, Oman \\ ${ }^{5}$ Department of Clinical Chemistry, College of Medical laboratory science, Gezira University, Sudan \\ ${ }^{6}$ Department of Medical Microbiology, College of Medical Laboratory Science, Gezira University, Sudan
}

\begin{abstract}
Background: In spite of all precautionary measurements have been done to control the spread of SARS-COV2, the mortality and morbidity have increased, and the need for an effective vaccine is growing daily

Aim: In this review, the authors provide an overview of the current COVID-19 vaccines under the spotlight.

Conclusion: Despite of the urgent need for a COVID-19 vaccine, several practical and technical issues remain, but recent vaccine developments by many companies and organizations seem promising and could make us closer to reduce mortality and morbidity rates.

KEYWORDS: Coronavirus; SARS-CoV-2; COVID-19; Vaccine; Immune response; Pandemic

ABBREVIATIONS: ARDS: Acute Respiratory Distress Syndrome; COVID-19: Corona Virus Disease of 2019; H1N: Influenza A virus subtype; MERS: Middle East Respiratory Syndrome; SARS: Severe Acute Respiratory Syndrome; RT-PCR: Real Time Polymerase Chain Reaction; APC: Antigen-presenting cell; ChAdOx1 nCOV-19; CH: Chimpanzee; AD: Adenovirus-Vectored Vaccine; Oxford Novel Coronavirus 2019; AZD1222: AstraZeneca Vaccines; CV: Coronavirus Vaccines; AD26: Adenovirus type 26; AD5: Adenovirus type 5; NOVAVAX: Nova Vaccines
\end{abstract}

\section{INTRODUCTION AND BACKGROUND}

Extreme acute respiratory distress syndrome (ARDS) caused by SARS-CoV-2 emerged as a local outbreak in Wuhan province at the end of December 2019, but later, when the disease spread to many countries worldwide, WHO declared SARS-CoV-2 as a pandemic disease called COVID-19 [1]. The SARS-CoV-2 genomic sequence analysis revealed its close resemblance to the SARS-COV and the Middle East Respiratory Syndrome coronavirus (MERS-COV) [2]. For the SARS-CoV-2 structure in Figure 1.
Quick Response Code:

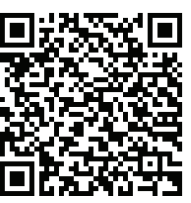

Address for correspondence: Nagia Suliman, Department of Clinical Chemistry, College of Medical laboratory science, Gezira University, Sudan

Received: January 30, $2021 \quad$ Published: February 05, 2021

How to cite this article: Ebtehal MF, Sheima. AE, Alaa AE, Ahmed OA, Abdelmonem MA, etc. al. COVID-19 and Promising Expected Vaccines. 2021- 3(1) OAJBS.ID.000253. DOI: $10.38125 / \mathrm{OAJBS} .000253$ 


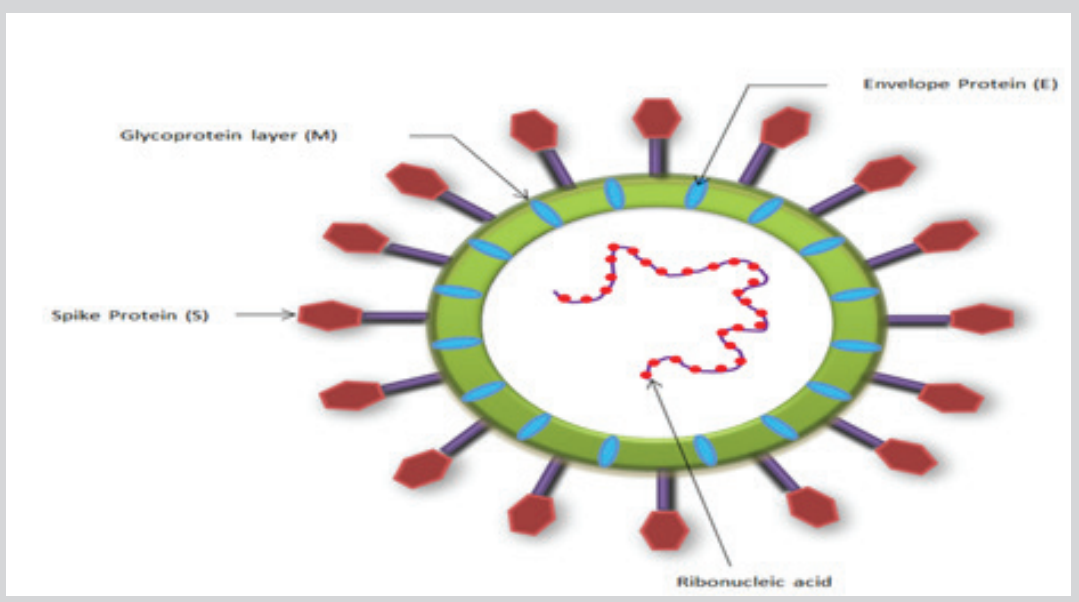

Figure 1: Illustrates the main structural features of Coronavirus SARS-CoV-2.

After identifying the genus of COVID-19, the world started its journey towards finding an ideal diagnostic tool for this disease, until reached that PCR is the standard golden method, at least up to date $[3,4]$. Then the journey is continuing to find out an efficient COVID-19 treatment strategy. Even though, the treatment line is unclear enough and representing a debated issue among medical communities, but it seems that the promising strategy against
COVID-19 is the vaccination process. The process of COVID-19 vaccines manufacturing has been approved by health authorities and organizations in many countries. Moreover, some countries have started the COVID-19 vaccine campaign for their citizens, such as the UK, USA and Russia. Herein, the narrative review provides an overview of the current COVID-19 vaccines under the spotlight.

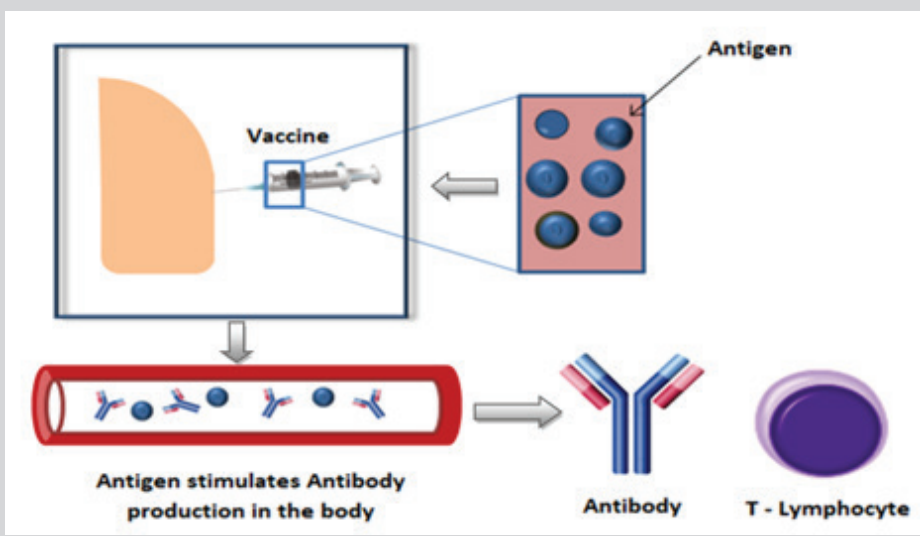

Figure 2: Schematic representing the administration of target antigen through a vaccine to stimulate the production of Antibodies and T- cell.

\section{CURRENT STATUS OF COVID-19 VACCINE}

A vaccine is a substance that stimulates the immune system to produce antibodies, precisely as if it was exposed to the disease [5] in Figure 2. Generally, vaccine is divided into different types, including inactivated vaccines, live attenuated vaccines, vectored vaccines (DNA/ RNA vaccines), nucleic acid-based vaccines, and recombinant subunit vaccines [6-9].

Currently, there are more than 300 COVID-19 vaccine candidates [10]. Most of those vaccine candidates are developed relied on the previous experience with other coronaviruses to prompt humoral antibodies against the viral particles such as spike protein or receptor binding domains [11]. The larger parts of COVID-19 vaccines are at the pre-clinical phase (phase I and II).

Phase I to investigate the vaccine safety in small group, then phase II to test the immunogenicity in large subjects and phase
III to estimate efficacy and safety of a vaccine to take decision for approval [12]. Currently, some of vaccines are authorized and recommended to prevent COVID-19.

\section{Pfizer Inc. and BioNTech}

Pfizer Company is a collaboration between Americans and Germans scientists through Pfizer Inc. and BioNTech SE. This collaboration led to innovate a vaccine candidate against COVID-19 [13]. Pfizer's vaccines based on using mRNA- candidate, which is considered as a new vaccines technique has never been approved in a vaccine before. According to Pfizer's announcement, the vaccines have demonstrated an efficacy rate of $95 \%$ after the first week of the second dose (3 weeks apart from the first dose) [14]. Data Monitoring Committee (DMC) has not raised any significant safety issues but has recommended that additional safety and efficacy data continue to be collected as planned and addressed consistently with regulatory authorities worldwide [15]. 
Regarding storage and distribution for vaccines, Pfizer's vaccines need to be stored in $-70{ }^{\circ} \mathrm{C}$ [16]. This frozen chain is going to be one of the most challenging aspects of the delivery of this vaccination because it will be difficult for rural areas and developing countries to access these types of ultra-cold freezers.

\section{Moderna's Vaccine (mRNA-1273)}

This type of Vaccine is collaboration between the National Institute of Allergy and Infectious Diseases and Moderna, Inc in USA [17]. Moderna's vaccine against COVID-19 relies on a novel technology that uses messenger RNA (mRNA) similar to Pfizer's vaccine which also depends on mRNA to express a protein called spike [18]. According to the developers of this type of vaccines say, one of the mRNA advantages is faster and simpler to manufacture than traditional vaccines, which can take time to develop because researchers have to grow and inactivate whole pathogens or their particles [16]. Even though, this novel mRNA vaccine might be more durable against pathogens that tend to mutate, such as coronaviruses, but still the instability and breaking down by nuclease enzymes are main concerns $[19,20]$.

The Moderna vaccine was $94.1 \%$ effective at blocking symptomatic Covid-19, measured starting from 14 days after the second dose (28 days from the first dose); [21]. It also has the same difficulty of storage in cold temperatures requires to be stored at $-20{ }^{\circ} \mathrm{C}[22]$.

\section{ChAdOx1 nCoV-19 Vaccine (AZD1222)}

ChAdOx1 nCoV-19 vaccine popularly known as the Oxford vaccine was developed by Oxford University in collaboration with the biopharmaceutical company AstraZeneca [23]. The Oxford vaccine is composed of a replication-deficient chimpanzee adenoviral vector ChAdOx1 carrying the SARS-CoV-2 structural surface glycoprotein antigen (spike protein; $\mathrm{nCoV}-19$ ) gene [23]. The mechanism of vaccine here based on inducing $\mathrm{T}$ cell lymphocytes within the first two weeks of vaccination and later after 28 days occurs humoral response (provoke of antibodies immunity) [24] Although the Oxford vaccine is a type of a weakened or Liveattenuated vaccines structure of a live virus, which can induce longterm of immune responses, however, this type of vaccines tends to be risky for those people with weakened immune systems or have other health issues [25].

\section{Sinovac's COVID-19}

CoronaVac is another type ofCOVID-19vaccines that synthesized by a Chinese company called Sinovac Biotech. The CoronaVac used a traditional inactivated virus technique (an inactivated version of the SARS-CoV-2 virus). This technique is relied on a quick triggering the immune system to produce a humoral response within 4 weeks of immunization by giving 2 doses of the vaccine at a 14- day [26]. Although CoronaVac produced levels of antibodies lower than those reported in people who had recovered from Covid-19, but the CoronaVac innovators believe it could still provide protection from the unprecedented coronavirus [27]. This lower level of antibodies demonstrates what was published in previous studies that, inactivated virus technique is considered as not fully protective as live vaccines and might require adjuvant over time [25]. One of Sinovac's main advantages is that CoronaVac could be stored at normal refrigerator at $2-8{ }^{\circ} \mathrm{C}$ [27]. Sinovac's COVID-19 vaccine has been approved by Chinese health authorities to use in emergency case $[28,29]$.

\section{Sputnik V}

The name of Sputnik came from the first Soviet space satellite. Sputnik V is the first vaccine against COVID-19 approved by Russian authorities. It's based on two vectors, which include adenovirus vector 1 AD26 and adenovirus vector 2 AD5 [30]. This is a novel vectors technique developed by Gamaleya center of Epidemiology and Microbiology. This technique is thought to produce a longerterm immune response as compared to the vaccines using one and the same vector. The Sputnik V is available in dry form (lyophilized), which makes it easier to storage $\left(2-8^{\circ} \mathrm{C}\right)$ and distributes [31].

\section{NOVAVAX}

NOVAVAX is an American company that has developed and is testing a candidate coronavirus vaccine called NVX-CoV2373. It's a type of nanoparticle vaccine consists of trimeric full-length SARS-CoV-2 spike glycoproteins and Matrix-M1 adjuvant [32]. The purpose of Matrix-M1 adjuvant is stimulating the entry of antigenpresenting cells (APC) into the injection site and thus enhancing the presentation of antigen to near lymph nodes, hence NVXCoV2373 induced humoral immune response that block attaching of spike protein to cellular receptors and provided immunity from infection [33]. The storage condition of NOVAVAX is convenient, it is transferred and handling in an unfrozen atmosphere, liquid formulation allows to be kept at $2{ }^{\circ} \mathrm{C}$ to $8{ }^{\circ} \mathrm{C}$, therefore for distribution could use standard vaccine channels [34].

\section{RE-INFECTION AND VACCINE}

The question of the possibility of reinfection occurrence after taking the vaccine still is remaining unclear. Nevertheless, if SARSCoV-2 follows the previous family of its coronavirus cousins, so, reinfection will soon become the rule, instead of the exception $[35,36]$. As it is seen in common-cold- to infect the same individual multiple times.

\section{VACCINE PRIORITY}

Nearly, most of the Committees that supervise on vaccination campaigns are almost agreed to divide the vaccines takers into Priority groups. For instance, a high risk of severe illness in highexposure occupations, Frontline health workers, and vulnerable people such as the eldest aged 70 years and over [37].

\section{TREATMENT}

So far, there is no unified approach to agreed-upon COVID-19 treatment. However, there is one international trial called Solidarity. It launched by the WHO and partners, aims to find out an effective treatment for COVID-19 [38]. This trial evaluated four types of treatments, Remdesivir, Hydroxychloroquine, lopinavir/Ritonavir and Interferon. It found those treatments had insignificant effect on overall mortality and hospitalized patients [39]. Only corticosteroids have proven to some extent effective against critical COVID-19 patients cases [40].

\section{QUESTIONS TO BE ADDRESSED}

There are some concerns among the public population about receiving COVID-19 vaccines. They are right toward these concerns because of the novelty and rapid development of it. Below are some questions that need convincing answers from the vaccine's developer. 
1- How is the vaccine safety was evaluated? People who are vaccinated for trials and followed up for time period, is this period sufficient to detect most safety issues?

\section{2- Will the Vaccine Be Effective for All Patients?}

3- Should Testing for COVID-19 continues after vaccination, and if so, for what duration?

4- Is there is a transparency and equality in COVID-19 distribution for those vulnerable groups?

\section{ACKNOWLEDGMENT}

Our great thanks to the Sudanese Medical Laboratory Technologist in Oman (SMLTO) for their logistic support.

\section{AUTHOR CONTRIBUTION}

Ebtehal M Fawzi, Sheima A Elbasheer, Alaa A Elhussein, conceptualization and wrote the original draft; Ahmed $\mathrm{O}$ Abu Elhassan, Nagia S Ahmed, Mayada K Ali Acquisition of data and designed; Abdelmonem M Ali, Alfatih Aboalbasher Yousif, reviewed the final draft. All authors approved the final version of the article.

\section{REFERENCES}

1. Ortiz-Prado E (2020) Clinical, molecular and epidemiological characterization of the SARS-CoV-2 virus and the Coronavirus Disease 2019 (COVID-19), a comprehensive literature reviews. Diagn Microbiol Infect Dis 98(1): 115094.

2. Naqvi AA (2020) Insights into SARS-CoV-2 genome, structure, evolution, pathogenesis and therapies: Structural genomics approach. Biochim Biophys Acta Mol Basis Dis 1866(10): 165878.

3. Wrapp D (2020) Cryo-EM structure of the 2019-nCoV spike in the prefusion conformation, Science. 367(6483): 1260-1263.

4. Ali AM, Alfatih A, Ahmed O (2020) Is a real time-polymerase chain reaction a reliable confirmatory test for COVID-19? Journal of Blood Disorders \& Transfusion 11(6): 442.

5. Centers for Disease Control and Prevention (CDC) (2012) Vaccines: The Basics.

6. Yu S, Gang W, Xiao PC (2020) An overview of COVID-19. J Zhejiang UnivSci B 21(5): 343-360.

7. Jieliang $W$ (2020) The COVID-19 vaccine race: Challenges and opportunities in vaccine formulation. AAPS Pharm SciTech 21(6): 225.

8. Van Riel D, de Wit E (2020) Next-generation vaccine platforms for COVID-19. Nature Materials 19: 810-820.

9. Wen-Hsiang Chen, Ulrich S, Peter JH (2020) The SARS-CoV-2 vaccine pipeline: An overview. Curr Trop Med Rep 7: 61-64.

10. Julia R (2020) The COVID-19 vaccines being backed by the UK and when we might get them. The Pharmaceutical Journal. 305: 7942.

11. Wangxue C (2020) Promise and challenges in the development of COVID-19 vaccines, Human Vaccines \& Immunotherapeutics 16(11): 2604-2608.

12. WHO (2004) Guidelines on clinical evaluation of vaccines: regulatory expectations. WHO technical report series. 924: 1.

13. Businesswire (2020) Pfizer and BioNTech announce vaccine candidate against COVID-19 achieved success in first interim analysis from phase 3 study. Businesswire.

14. Zimmer C, Corum J, Lee WS (2021) COVID-19 vaccine tracker: What's the status of all vaccines in development? New York times.

15. Biontech de (2020) Pfizer and BioNTech announce vaccine candidate against COVID-19 achieved success in first interim analysis from Phase 3 study. investors.biontech.de.
16. Yasemin S (2020) Here are the most promising coronavirus vaccine candidates out there. Live Science.

17. Shi Y, Wang N, Zou QM (2020) Progress and challenge of vaccine development against 2019-novel coronavirus (2019-nCoV). Zhonghua Yu Fang Yi Xue Za Zhi 54(6): 614-619.

18. Jon C (2020) Absolutely remarkable: No one who got moderna's vaccine in trial developed severe COVID-19. Science.

19. Simran PK, Vandana G (2020) COVID-19 Vaccine: A comprehensive status report. Virus Research 288: 198114.

20. Abishek W, Anas A, Abhijeet L, Camilla F, Aneesh T, et al. (2020) Opportunities and challenges in the delivery of mRNA-based vaccines. Pharmaceutics 12(2): 102.

21. Lisa A Jackson, Evan J Anderson, Nadine G Rouphael, et al. (2020) An mRNA Vaccine against SARS-CoV-2 Preliminary Report. New England Journal of Medicine. 383(20): 1920-1930.

22. Abhishek De (2020) Explained: How effective are the top COVID-19 vaccines, and when will they be available? Indian Express.

23. Voysey, Merryn Aban, Marites (2020) Safety and efficacy of the ChAdOx1 nCoV-19 vaccine (AZD1222) against SARS-CoV-2: an interim analysis of four randomized controlled trials in Brazil, South Africa, and the UK. The Lancet 0(0): 1-13.

24. Oxford University (2020) New study reveals Oxford coronavirus vaccine produces strong immune response. Oxford University's Jenner Institute.

25. US Department of Health \& Human Services (2020) Vaccine types. HHS GOV.

26. Yanjun Z, Gang Z, Hongxing P (2020) Safety, tolerability, and immunogenicity of an inactivated SARS-CoV-2 vaccine in healthy adults aged 18-59 years: a randomized, double-blind, placebo-controlled, phase 1/2 clinical trial. Lancet Infect Dis 1-12.

27. Robert H (2020) Sinovac's Covid-19 vaccine appears safe and prompts quick immune response study finds. Forbes.

28. Miyoung K, Roxanne L (2020) Sinovac's COVID-19 vaccine induces quick immune response- study. Reuters.

29. Roxanne L, Tony M (2020) Exclusive: $90 \%$ of China's sinovac employees, families took coronavirus vaccine, says CEO. Reuters.

30. Sputnik V (2020) General information - Sputnik V.

31. Robert C, Kelley L (2021) Sputnik V vaccine. Precision vaccinations.

32. Cheryl K, Gary A, Iksung C (2020) Phase 1-2 Trial of a SARS-CoV-2 recombinant spike protein nanoparticle vaccine. New England Journal of Medicine 383(24): 2320-2332.

33. Gaithersburg (2020) Novavax announces COVID-19 vaccine clinical development progress. Globe Newswire.

34. Gaithersburg (2020) Novavax initiates phase 3: Efficacy trial of COVID-19 vaccine in the United Kingdom. Globe Newswire.

35. William AH (2020) What COVID-19 reinfection means for vaccines.

36. Iwasaki A (2021) What reinfections mean for COVID-19. The Lancet. Infectious diseases 21(1): 3-5.

37. JCVI (2020) Joint committee on vaccination and immunization: advice on priority groups for COVID-19 vaccination.

38. Pan H, Peto R, Henao Restrepo AM (2020) Repurposed antiviral drugs for Covid-19 - Interim WHO solidarity trial results. N Engl J Med: oa2023184.

39. Owen D (2020) Covid-19: Remdesivir has little or no impact on survival, WHO trial shows. BMJ 371: m4057.

40. WHO (2020) Corticosteroids for COVID-19. 